\title{
Le aggressioni nei confronti degli infermieri dei dipartimenti di emergenza: revisione della letteratura internazionale
}

\author{
Aggressions towards nurses in emergency departments: an international literature review
}

Lorenzo Brunetti1 Stefano Bambi2

\begin{abstract}
RIASSUNTO
Il fenomeno delle violenze sul luogo di lavoro è presente in tutti i contesti lavorativi. Tra questi figurano i dipartimenti di emergenza (DEA), caratterizzati da un ampio numero di accessi quotidiani, da situazioni ad alto contenuto emotivo, e da stress legato alla gestione delle priorità diagnostico-terapeutico-assistenziali. Allo scopo di quantificare il fenomeno delle aggressioni verso gli infermieri dei pronto soccorso, discriminarne tipologie ed perpetratori, ed identificare le conseguenze a livello individuale e organizzativo, è stata condotta una revisione di letteratura sui database Medline, CINHAL, e Medscape. 35 articoli originali inclusi, tra cui è stato possibile reperirne 29. La frequenza degli abusi verbali riportati dagli infermieri operanti nei DEA oscilla tra il $50 \%$ ed il $100 \%$ degli intervistati nei vari studi, mentre la violenza fisica viene riportata in quote tra il $16.7 \%$ ed il $72 \%$. Pazienti e familiari sono ritenuti i principali autori di aggressioni, seguiti in minor parte dai medici, e, solo in basse percentuali, da colleghi di pari grado. L'abuso di alcool, sostanze stupefacenti e l'overcrowding in DEA sono fattori scatenanti degli eventi di violenza. L'under-reporting degli episodi di aggressione è frequente fino all' $80 \%$ delle vittime, e da alcuni lavori emerge che le aggressioni sono vissute come parte del normale lavoro. E' stata dimostrata una correlazione diretta tra aggressioni e sintomi di disturbo da stress post-traumatico. Inoltre è riportato anche il possibile abbandono del lavoro. Eventi formativi mirati sembrano efficaci per la diminuzione del numero di violenze, e per l'adozione di comportamenti adattivi.
\end{abstract}

Parole Chiave: aggresione, emergenza, violenza, luogo di lavoro.

ABSTRACT

Workplace violence is a widespread phenomenon in every kind of settings. Among these ones there are emergency departments (ED), that have distinctive features as like the large daily number of patients' presentations, and high emotional content or stressing situations related to the management of diagnostic-therapeutic priorities. We reviewed the medical and nursing literature to quantify the international widespread of aggressions towards nurses working in EDs, distinguish the typologies and the perpetrators, and identify the consequences on victims and healthcare organizations. Original papers were searched using Medline, CINHAL, and Medscape databases. 35 research articles met the inclusion criteria, but 6 were not retrieved. The rate of verbal abuses reported by ED nurses varies from $50 \%$ to $100 \%$ of those who were surveyed, while physical violence ranges between $16.7 \%$ and $72 \%$. Patients and relatives are the main perpetrators, followed by doctors, and, only in lower percentages, by nurses colleagues. Alcohol, drugs abuse, and overcrowding in EDs are acknowledged as motivating factors for violent events. Underreporting of aggressions is frequent up to the $80 \%$ of victims, and some papers report that nurses consider assaults as a normal part of their work. There is a direct relation between aggressions and symptoms of post-traumatic stress disturb syndrome. Moreover there is a sense of continuous fear in nurses, causing the likelihood of workplace leaving. Special educational courses seem to be effective in diminishing the number of aggressions and to adopt adequate adaptive behaviors.

Key words: aggression emergency nursing, violence, workplace

INTRODUZIONE

$\mathrm{L}$ a violenza sul luogo di lavoro è un fenomeno ormai da molti anni ampiamente documentato. Di fatto, sono molteplici le definizioni offerte da diversi Enti e Associazioni internazionali che si occupano del

1 Infermiere, Terapia Intensiva di Emergenza - DAI DEA, Azienda Ospedaliero Universitaria Careggi, Firenze

2 Infermiere, Terapia Intensiva di Emergenza - DAI DEA, Azienda Ospedaliero Universitaria Careggi, Firenze - Dottorando di Ricerca in Scienze Infermieristiche - Università degli Studi di Firenze Corrispondenza: stebam@hotmail.it problema (CDC Workplace Safety and Health, 2002; Department of Health Western Australia, 2004), tra cui la più completa è forse quella fornita dalla Occupational Safety \& Health Administration (OHSA), che la descrive come "qualsiasi atto o minaccia di violenza fisica, molestie, intimidazioni, o altri comportamenti di minaccia che si verifichino sul posto di lavoro. Si va da minacce e abusi verbali ad aggressioni fisiche e perfino omicidi. Può interessare e coinvolgere i dipendenti, clienti e visitatori" (United States Department of Labor, 2012). L'effetto di queste azioni si ripercuote 
non solo sulle vittime, ma anche sui testimoni e persino su chi si senta in essa coinvolto come membro di un gruppo al cui interno siano state subite aggressioni. Si genera di conseguenza un clima di sfiducia, di ostilità e di paura, e vengono meno le basi del lavoro di gruppo e della condivisione degli obiettivi, ripercuotendosi sull'operato dei singoli e sulla qualità delle cure prestate dal gruppo (Perrone, 1999). In conseguenza a questo gli episodi di violenza sul luogo di lavoro devono essere considerati dei veri e propri eventi sentinella: indici di situazioni di rischio che richiedono l'adozione di opportune ed immediate misure di protezione della salute degli operatori (The Joint Commission, 2010).

Per quanto concerne la realtà italiana, gli "atti di violenza a danno di operatore" sono riconosciuti ormai da diversi anni come evento sentinella (Ministero della Salute, 2009), e sono stati oggetti di opportune raccomandazioni per la loro prevenzione (Ministero della Salute, 2007).

Benché il fenomeno delle violenze sul luogo di lavoro sia sostanzialmente distribuito su tutti i contesti lavorativi, l'ambito sanitario presenta alcuni ambiti particolarmente critici. Tra questi figurano i dipartimenti di emergenza (DEA), che sono caratterizzati da un ampio numero di accessi quotidiani, e da situazioni ad alto contenuto emotivo, dove gli operatori devono muoversi mantenendo i difficili equilibri tra le necessità di assegnare priorità ai loro interventi, di agire rapidamente sotto il profilo diagnostico-terapeutico-assistenziale, e di garantire comunque una risposta sanitaria di qualità, mantenendo sempre elevato il profilo di umanizzazione delle cure. L'infermiere di pronto soccorso (PS), la cui attività di front office e quella di interfaccia tra i servizi dell'ospedale è centrale, sempre più frequentemente sale agli onori delle cronache per gli episodi di violenza nei quali si trova coinvolto durante lo svolgimento del proprio lavoro.

Pertanto, allo scopo di quantificare a livello nazionale ed internazionale il fenomeno delle aggressioni verso gli infermieri che operano pronto soccorso, discriminarne le tipologie ed i perpetratori, ed identificare quali conseguenze abbiano le violenze a livello individuale e delle organizzazioni di lavoro, è stata condotta la presente revisione di letteratura.

\section{METODO}

La ricerca bibliografica è stata compiuta esclusivamente sul web, avvalendosi di 2 banche dati ed un sito internet specialistico: Pubmed, il Cumulative Index to Nursing and Allied Health Literature (CINAHL), e Medscape. Sono state utilizzate le seguenti parole chiave combinate diversamente tra loro: "violence", “violent", "aggression", “abuse", "aggressive”, "emergency department", "nurses". I limiti impostati sulle ricerche sono stati relativi alla lingua italiana e inglese.

\section{RISULTATI}

Dopo i vari passaggi di analisi e selezione dei record reperiti attraverso $i$ database consultati, sono stati inclusi nella revisione 29 articoli di ricerca originali (Figura 1).

La varietà geografica di provenienza dei lavori pubblicati in letteratura è indicativa di quanto il problema delle aggressioni nei confronti degli infermieri di PS sia diffuso e globalmente percepito (Figura 2). La maggioranza degli studi viene dal territorio australiano, seguita da Stati Uniti, Irlanda, Gran Bretagna, Cina, Iran, Italia e Turchia. I disegni di studio prevalentemente impiegati nella letteratura sul tema sono di tipo descrittivo ed analitico, frequentemente con metodo misto qualitativo/quantitativo, attraverso un ampio uso di questionari (ben 19 su 29 lavori pubblicati).

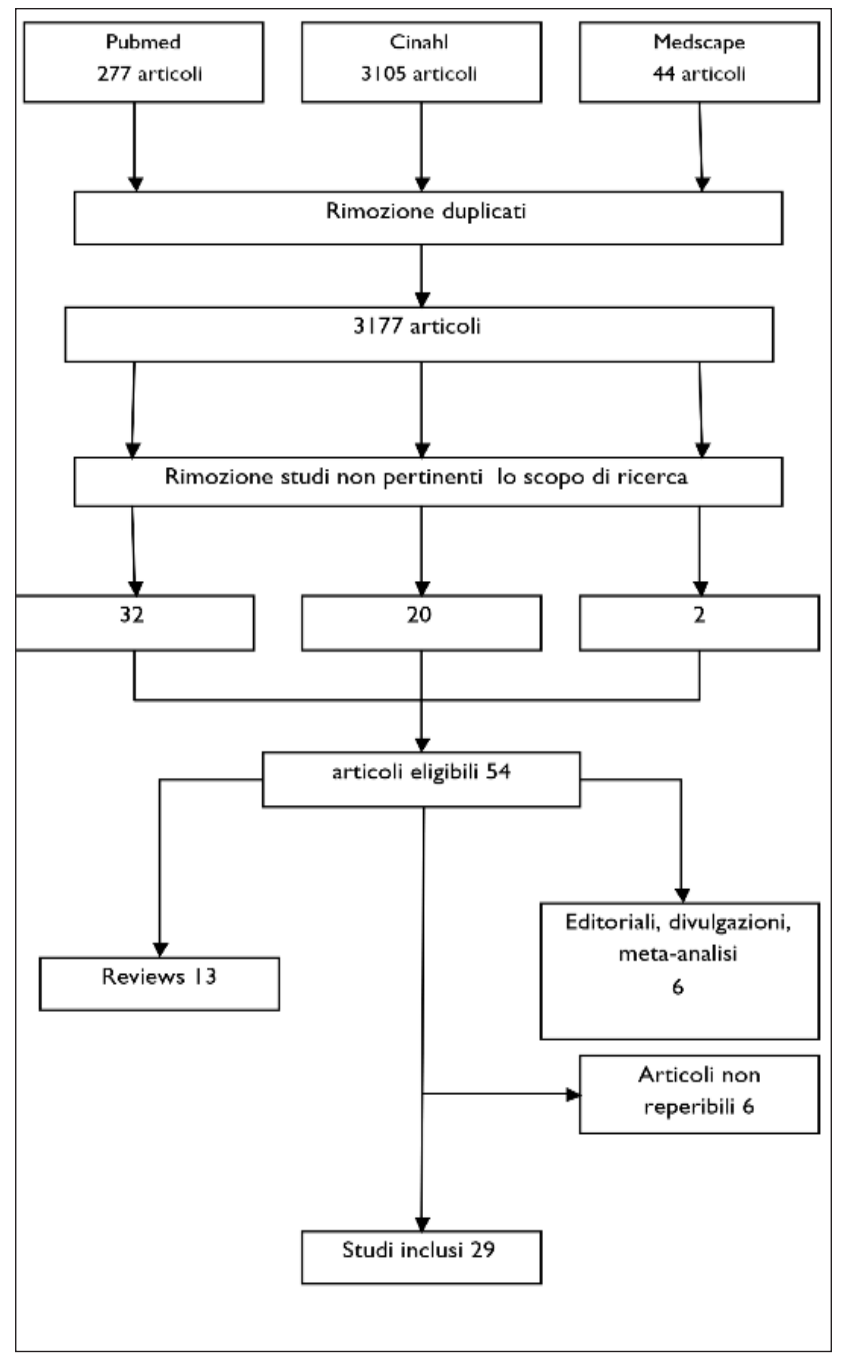

Figura 1 - Sintesi dei risultati della ricerca bibliografica 


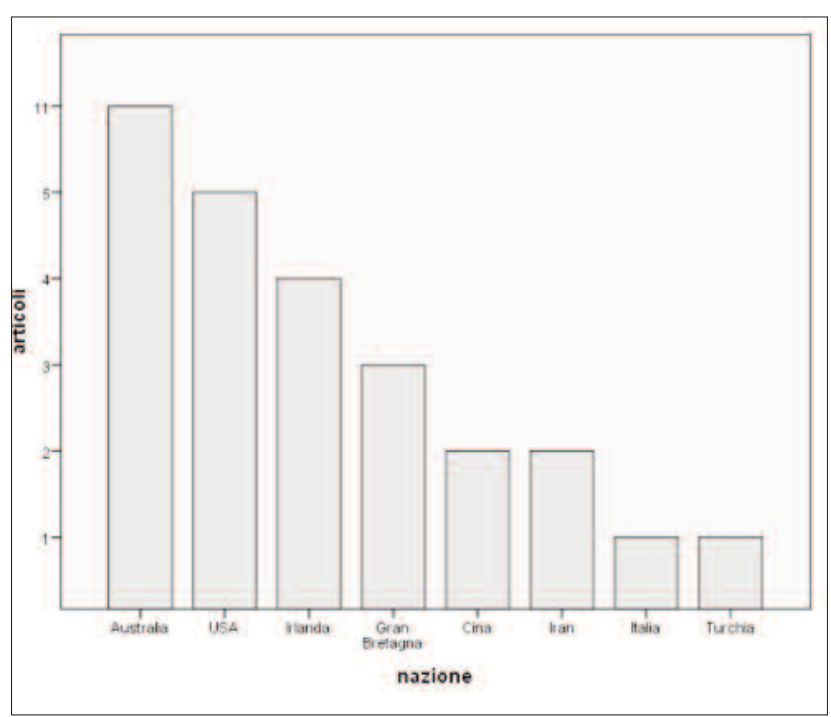

Figura 2 - Provenienza geografica dei lavori di ricerca

Prevalenza del fenomeno delle aggressioni e descrizione dei perpetratori

Il fenomeno della violenza nei DEA risulta ampiamente diffuso a livello mondiale. In Tabella 1 si riportano le quote di violenza riportate dai singoli studi, accanto alle tipologie di aggressori, dove descritte.

Le percentuali più elevate di infermieri vittime di aggressioni verbali si registrano negli studi di Deans (2004) e di Lyneham (2000), entrambi condotti in Australia, in cui il $100 \%$ degli infermieri dichiaravano di essere stati vittima di aggressioni verbali. Le quote più basse sono invece state riportate da Gillespie et al. (2010) e da Crilly e collaboratori (2004). $\mathrm{Nel}$ primo studio emerge che il $50 \%$ di un gruppo composto da 31 tra medici, infermieri e assistenti di un DEA pediatrico, è stato vittima di attacchi verbali. Nel secondo, la percentuale di aggressioni verbali subite è del 53\% in un gruppo di 71 infermieri di un PS australiano.

Per quanto concerne il panorama italiano, Zampieron et al. (2010), nel loro studio effettuato su 595 infermieri, rilevano quote di violenze verbali pari all' $82 \%$. Risultati che concordano con quelli della survey non pubblicata realizzata da Becattini et al. (2007) su un campione di 308 infermieri da svariati DEA italiani, in cui i tassi di aggressione verbale raggiungevano il $90 \%$.

Distinguendo tra abusi verbali e fisici, si denota come la maggioranza delle ricerche riportino alti tassi di aggressioni verbali, che vanno dal 73\% (Kwok et al., 2006) al 90\% (Gilchrist et al., 2011), mentre, per quanto concerne gli episodi di violenze fisiche, la variabilità dei risultati si presenta molto più ampia.

Pinar et al. (2011), in Turchia, hanno registrato la percentuale più importante di violenze fisiche, ovvero il $74,9 \%$ su di un campione di 255 infermiere. Anche

\begin{tabular}{|c|c|c|}
\hline Autori & $\begin{array}{c}\text { Tipologie di } \\
\text { violenza/abuso }\end{array}$ & Perpetratori \\
\hline Adeb-Saeedi (2002) & $\begin{array}{l}\text { Verbale } 96 \% \\
\text { Fisica } 70 \%\end{array}$ & Non indicato \\
\hline Cahill (2008) & $\begin{array}{l}\text { Verbale77\% } \\
\text { Fisica } 35 \%\end{array}$ & Non indicato \\
\hline Crabbe et al. (2002) & $\begin{array}{l}\text { Verbale } \\
\text { Fisica } 72 \%\end{array}$ & Non indicato \\
\hline Crilly et al. (2004) & $\begin{array}{l}\text { Verbale53\% } \\
\text { Fisica } 26 \%\end{array}$ & Pazienti \\
\hline Deans (2004) & $\begin{array}{l}\text { Verbale } 100 \% \\
\text { Fisica } 34 \%\end{array}$ & Non indicato \\
\hline Eslamian et al. (2010) & $\begin{array}{c}\text { Verbale } 68,2 \% \text { (media) } \\
\text { Fisica } 16,7 \% \text { (media) }\end{array}$ & Non indicato \\
\hline Gilchrist et al. (2011) & $\begin{array}{l}\text { Verbale } 90 \% \\
\text { Fisica } 25 \%\end{array}$ & $\begin{array}{c}\text { Pazienti } \\
\text { 91\%Accompagnatori } \\
61 \%\end{array}$ \\
\hline Gillespie et al. (2010) & $\begin{array}{l}\text { Verbale } 50 \% \\
\text { Fisica } 50 \%\end{array}$ & $\begin{array}{c}\text { Familiari } 82 \% \\
\text { verbale; } 24 \% \text { fisica } \\
\text { Pazienti } 18 \% \\
\text { verbale; } 76 \% \text { fisica }\end{array}$ \\
\hline Kwok et al (2006) & $\begin{array}{l}\text { Verbale } 73 \% \\
\text { Fisica } 18 \% \\
\text { Sessuale } 12 \%\end{array}$ & $\begin{array}{c}\text { Pazienti } \\
\text { Familiari } \\
\text { Medici } \\
\text { Colleghi }\end{array}$ \\
\hline Jenkins et al. (1998) & $\begin{array}{l}\text { Verbale } \\
\text { Fisica } 90 \%\end{array}$ & $\begin{array}{c}\text { Pazienti } \\
\text { Accompagnatori } \\
\text { Parenti }\end{array}$ \\
\hline Lyneham (2000) & $\begin{array}{l}\text { Verbale } 100 \% \\
\text { Fisica } 89 \%\end{array}$ & $\begin{array}{l}\text { Pazienti } 92 \% \\
\text { Parenti } 92 \% \\
\text { Medici } 26 \% \\
\text { Colleghi } 21 \%\end{array}$ \\
\hline Opie et al. (2010) & $\begin{array}{c}\text { Verbale } 79,5 \% \\
\text { Fisica } 28,6 \% \\
\text { Sessuale22,5\% } \\
\text { Stalking 4,9\% }\end{array}$ & Non indicato \\
\hline Pich et al. (2009) & $\begin{array}{c}\text { Verbale } 85 \% \\
\text { Fisica non indicato }\end{array}$ & Non indicato \\
\hline Pinar et al. (2010) & $\begin{array}{l}\text { Verbale } 91,4 \% \\
\text { Fisica } 74,9 \%\end{array}$ & $\begin{array}{c}\text { Accompagnatori } 45,5 \% \\
\text { Pazienti } 9,8 \%\end{array}$ \\
\hline Rose (1997) & $\begin{array}{l}\text { Verbale } 52 \% \\
\quad \text { Fisica }\end{array}$ & Non indicato \\
\hline \multirow[t]{2}{*}{ Ryan et al. (2006) } & \multirow{2}{*}{$\begin{array}{c}\text { Verbale } 89,2 \% \\
\text { Fisica } 22,9 \% \\
\text { Sessuale } 8,6 \%\end{array}$} & $\begin{array}{l}\text { Pazienti e familiari } \\
75,8 \% \text { verbali }\end{array}$ \\
\hline & & $75 \%$ fisiche \\
\hline Schnieden et al. ( 1995) & $\begin{array}{c}\text { Verbale } 86,6 \% \\
\text { Fisica34,6\% }\end{array}$ & Non indicato \\
\hline Tang et al. (2007) & $\begin{array}{c}\text { Verbale } 91,5 \% \\
\text { Fisica } 29,7 \%\end{array}$ & $\begin{array}{c}\text { Familiari } 81,5 \% \\
\text { Pazienti } 75,9 \% \\
\text { Medici } 42,1 \% \\
\text { Colleghi } 15,3 \%\end{array}$ \\
\hline $\begin{array}{l}\text { Winstanley et al. } \\
\text { (2004) }\end{array}$ & $\begin{array}{l}\text { Verbale } 75 \% \\
\text { Fisica } 30,8 \%\end{array}$ & $\begin{array}{l}\text { Pazienti } 26,9 \% \text { fisica. } \\
\text { Visitatori } 5 \% \text { fisica. }\end{array}$ \\
\hline Zampieron et al. (2010) & $\begin{array}{l}\text { Verbale } 71 \% \\
\text { Fisica } 29 \%\end{array}$ & $\begin{array}{c}\text { Pazienti e accompagna- } \\
\text { tori } 58,3 \% \\
\text { Colleghi } 21 \%\end{array}$ \\
\hline
\end{tabular}

Tabella 1 - Prevalenza delle aggressioni e tipologie di perpetratori 
nello studio del 2002 effettuato da Crabbe et al. (2002), condotto in Irlanda, il tasso è simile, attestandosi al $72 \%$ di 156 infermieri. Di contro, Eslamian et al. (2010) e Kwok et al. (2006), riportano le quote minori di violenza fisica sinora registrate, rispettivamente del $16,7 \%$ e $18 \%$.

In Italia, Becattini et al. (2007) rilevano aggressioni fisiche nel il 35\% degli infermieri rispondenti al questionario, mentre nello studio di Zampieron et al. (2010) questa percentuale scende al 29\%.

In generale, la violenza fisica sembra comunque avere un impatto minore a livello di frequenza, rispetto a quella verbale.

Per quanto riguarda i perpetratori delle violenze fisiche e verbali, nonostante la larga mancanza di dati, ci sono dei risultati estremamente significativi. Nello studio di Gilchrist et al. (2011), i pazienti sono ritenuti responsabili del 91\% delle aggressioni fisiche, anche se questo numero può essere difficilmente paragonato agli altri, in quanto ricavato in un DEA esclusivamente pediatrico. Lyneham (2000), dall'analisi di 266 questionari di infermieri, rilevano che i pazienti sono responsabili del $92 \%$ delle violenze fisiche e che ai familiari dei pazienti viene attribuito il $92 \%$ di tutte le aggressioni. Nello studio di Tang et al. (2007), i familiari vengono indicati come i principali perpetratori di aggressioni verbali $(81,5 \%$ degli infermieri rispondenti), in linea con il rilievo di Gillespie et al. (2010) che riportano una percentuale del $82 \%$.

\section{Fattori di rischio e fattori precipitanti degli episodi di violenza}

Sembra che alcool e stupefacenti siano molto spesso alla base di episodi di aggressività verso gli operatori dei PS: nella ricerca di Crilly et al. (2004), gli autori delle aggressioni erano percepiti sotto l'influenza di alcool e sostanze stupefacenti nelle percentuali del $30 \%$ e del $25 \%$. L'alcool viene imputato come fattore scatenante per le aggressioni dal 78\% degli infermieri australiani (Gilchrist et al. 2011), mentre l'uso di stupefacenti è visto come causa di violenza dal 70\%. Queste percentuali salgono ulteriormente nello studio di Jenkins et al. (1998), in cui il $98 \%$ di 219 consulenti medici a capo di altrettanti dipartimenti di emergenza, ritiene l'alcool uno dei maggiori fattori precipitanti per le situazioni a rischio di violenze, seguito dall'utilizzo di droghe. Inoltre, dal momento che nell'area triage che nelle stanze visita, non esistono barriere di alcun genere tra operatori ed utenza, questa condizione pone gli infermieri e i medici maggiormente a rischio di violenze fisiche, a differenza degli addetti all'accoglienza, che stando dietro ad un vetro risultano maggiormente sottoposti ad aggressioni verbali (Jenkins et al. 1998). Pertanto, nelle succitate aree del DEA risultano un maggior numero di aggressioni (Crilly et al. 2004; Ministero della Salute, 2007; Becattini et al. 2007).

Nelle ricerche esaminate l'overcrowding, che è un problema diffuso a livello internazionale (Bambi et al. 2011), viene percepito come una delle cause scatenanti per le aggressioni.

Nello studio di Tang et al. (2007), infatti, il sovraffollamento è considerato come la prima causa di aggressioni verso gli operatori per l'89\% degli infermieri, mentre nei lavori di Lyneham (2000) e di Jenkins et al. (1998) viene indicato come seconda causa rispettivamente dall' $85 \%$ e $86 \%$ dei rispondenti. Questi risultati sono confermati anche per la realtà italiana in cui, dalle dichiarazioni degli infermieri emerge che gli aggressori più comuni sono proprio i pazienti stanchi di aspettare, seguiti da parenti ed accompagnatori (Becattini et al. 2007).

\section{Violenza proveniente dall'interno del gruppo di lavoro}

Le aggressioni cui gli infermieri possono andare incontro non sono esclusivo appannaggio di coloro che arrivano in DEA in veste di utenti. Secondo lo studio effettuato da Tang et al. (2007), gli abusi verbali sono da imputare anche ai medici per il $42,1 \%$ e ai colleghi infermieri per il $15,3 \%$. Le violenze fisiche vengono attribuite allo staff medico per il $4,3 \%$ mentre non se ne ascrivono ai colleghi di pari ruolo. Anche Kwok et al. (2006), pur non indicando le percentuali precise, annoverano tra i perpetratori anche i medici e infermieri. Nell'analisi effettuata da Lyneham (2000) risulta che i medici sono responsabili del $26 \%$ delle aggressioni e gli infermieri del $21 \%$. La stessa percentuale viene rilevata anche sul suolo nazionale da Zampieron et al. (2010).

\section{Le vittime delle aggressioni}

Tra gli studi analizzati in questa revisione, solo quattro descrivono le caratteristiche peculiari delle vittime di aggressione (Tabella 2). Da questi si evidenzia che mentre le donne sono più colpite da abusi verbali, gli uomini si trovano più spesso coinvolti in violenze di tipo fisico (Gillespie et al. 2010). Considerando invece la macro-categoria delle violenze in generale, lo studio italiano di Zampieron et al. (2010), si pone in lieve controtendenza rispetto ai rilievi di Gilchrist et al. (2011) e Kwok et al. (2006), in cui la percentuale delle aggressioni verso gli uomini risulta più elevata di quella delle donne.

L'"identikit" della figura più a rischio di aggressioni sembra essere quella delle infermiere dei DEA (Zampieron et al. 2010), con un'esperienza lavorativa 


\begin{tabular}{|c|c|c|c|c|}
\hline Autore & Campione & $\begin{array}{c}\text { Rapporto } \\
\text { maschi/ } \\
\text { femmine }\end{array}$ & $\begin{array}{c}\text { Violenze subi- Violenze subi- } \\
\text { te da uomini }\end{array}$ & te da donne \\
\hline Cahill (2008) & 65 & $1: 6$ & Verbali 11\% & Verbali 25\% \\
\cline { 4 - 5 } & & Fisiche 33\% & Fisiche 13\% \\
\hline $\begin{array}{c}\text { Gilchrist et al. } \\
\text { (2011) }\end{array}$ & 91 & $4: 5$ & $88.20 \%$ & $47.10 \%$ \\
\hline $\begin{array}{c}\text { Kwok et al. } \\
\text { (2006) }\end{array}$ & 420 & $1: 10$ & 88 & 75 \\
\hline $\begin{array}{c}\text { Zampieron } \\
\text { et al. (2010) }\end{array}$ & 595 & $1: 4$ & $42 \%$ & $52 \%$ \\
\hline
\end{tabular}

Tabella 2 - Percentuali di violenze subite dagli infermieri di PS divisi per genere

all'interno del dipartimento in media di 5 anni (Zampieron et al. 2010; Rose, 1997), una laurea (Zampieron et al. 2010) o una formazione riconosciuta come " middle grade" (Winstanley et al. 2004), e con età dai 31 ai 40 anni (Zampieron et al. 2010).

\section{Le denunce e segnalazioni degli episodi di violenza subiti}

Il fenomeno delle aggressioni sul luogo di lavoro a carico degli infermieri di pronto soccorso sembra essere gravato da una diffusa prassi di non segnalazione degli episodi subiti.

Un ampio numero di studi riporta che le aggressioni, specialmente quelle verbali, vengono difficilmente denunciate dagli operatori. In una ricerca si riportano livelli di denuncia abituali solo del 14\% (Lyneham, 2000), e sono poche le realtà in cui le aggressioni vengono segnalate in numero congruo, pari al 60\% (Tang et al. 2007), o fino al 70\% (Crilly et al. 2004).

Nello studio di Pinar et al. (2011), l'80\% degli infermieri vittima di violenze dichiara di non denunciare le aggressioni subite e ne attribuisce la principale motivazione ad una mancanza di soddisfazione nelle risposte fornite dall'amministrazione.

L'under-reporting del fenomeno sembra essere dovuto anche al fatto che le vittime considerano meno grave l'aggressione subita durante il lavoro rispetto al medesimo incidente occorso ad un privato cittadino, quasi che le aggressioni facessero parte del normale lavoro degli infermieri (Eslamian et al. 2010; Rose, 1997). Per alcuni il fenomeno è diventato così comune da essere parte integrante del lavoro (Crilly et al. 2004; Pich et al. 2011). Altri motivi per cui le denunce non vengono fatte sono la paura del giudizio dei colleghi, o per vergogna (Crilly et al. 2004; Luck et al. 2008).

\section{Reazioni delle vittime}

Diverse ricerche tra quelle prese in esame, hanno cercato di correlare il fenomeno delle aggressioni con le conseguenze psicologiche e lavorative. Nello studio di Opie et al. (2010), viene dimostrata una correlazione diretta tra aggressioni e sintomi di disturbo da stress post-traumatico (PTSD) e come le aggressioni generino un senso di continua paura nel personale, che sarebbe tra le prime cause di abbandono del lavoro, come riportato anche nello studio di Gilchrist et al. (2011), nel quale si rileva anche un aumento nei costi delle cure dovuti all'assenza da lavoro per malattia del personale coinvolto in violenze. Il sentimento di continua paura viene segnalato anche nel lavoro svolto da Pinar et al. (2011), in cui il 65\% degli infermieri afferma di provare una continua angoscia di essere di nuovo vittima di violenza.

Gates et al. (2011) nella loro analisi, hanno valutato quanto risultassero psicologicamente colpiti gli operatori da una violenza e quanto ne fosse compromessa la performance lavorativa successiva. Dai dati raccolti emerge che il 94\% del personale mostrava la presenza di almeno un sintomo di stress, e, cosa ancora più grave, il $17 \%$ aveva raggiunto livelli tali da poter essere considerata la diagnosi di PTSD. Inoltre le performance sia cognitive che relazionali del personale risultavano chiaramente diminuite, tanto da arrivare ad affermare che gli infermieri sottoposti a violenze avevano problemi a rimanere cognitivamente ed emozionalmente concentrati nelle loro attività lavorative, anche se affermavano il contrario. Gillespie et al. (2010) aggiunge, inoltre, che il fenomeno della violenza genera nelle strutture un caos che può causare ritardi nelle cure agli altri pazienti e fornire una pessima immagine dell'ospedale e degli operatori.

Luck et al. (2008) hanno cercato di investigare quali significati gli infermieri attribuiscano alle aggressioni e come reagiscano a queste. Gli infermieri arrivano a discriminare le diverse forme di violenza in una maniera molto complessa rispetto alle altre persone. Viene vista in modo differente la violenza verso gli operatori, se considerata come simbolo del sistema organizzativo o se è diretta verso l'operatore come persona. Le risposte alle aggressioni e le considerazioni sulla violenza cambiano anche se ad analizzare l'evento è la vittima oppure un testimone. Gli infermieri, inoltre, individuano una serie di fattori che fungono da attenuanti sulle responsabilità di violenze. Questi fattori rientrano nelle categorie sociali, psicologiche, organiche, psichiatriche, e mediche.

Una analisi più particolareggiata proviene dallo studio di Tang et al. (2007), nel quale il 44,3\% delle vittime si è sentito offeso, il $75,7 \%$ ha dichiarato che il proprio morale è calato ed il $58,6 \%$ ha affermato 
che la qualità del proprio operato ne è risultata colpita.

Altri studi invece hanno valutato la percezione di sicurezza che gli operatori hanno all'interno delle loro strutture lavorative, dimostrando che la categoria degli infermieri è quella che si sente meno al sicuro nei DEA (Kansagra et al. 2008). Infatti, il 65\% degli infermieri non si sente mai al sicuro (Pinar et al. 2011), sono più a rischio di violenza quando sono stanchi, stressati, demotivati, e queste situazioni sembrano essere causate dall'organizzazione del lavoro (Zampieron et al. 2010).

Alcuni infermieri in seguito alle violenze subite hanno messo in campo strategie di coping diverse come: shopping, preghiere o tentativi di vendetta. In seguito alle violenze verbali e fisiche subite, è stato riportato addirittura un caso di tentato suicidio (Kwok et al. 2006).

\section{Soluzioni al problema}

In molti lavori pubblicati, gli infermieri intervistati denunciano una formazione sulla gestione delle situazioni di rabbia e violenza che in molti casi risulta molto carente (Pinar et al. 2011; Kansagra et al. 2008; Ryan et al. 2006). Ne consegue un desiderio da parte degli operatori di migliorare le loro competenze o di aggiornarle. Alcuni studi (Eslamian et al 2010; Deans, 2004) dimostrano chiaramente come anche semplici giornate formative possano ottenere ottimi risultati nella diminuzione del numero di aggressioni (Eslamian et al 2010; Deans, 2004), nel produrre la sensazione negli operatori di essere in grado di affrontare situazioni difficili (Deans, 2004), e nel fornire gli strumenti adatti per riuscire a comprendere e adottare i giusti comportamenti di fronte ad atteggiamenti aggressivi (Eslamian et al 2010). Gli operatori, dopo essere stati adeguatamente istruiti ed informati, riportano un maggior senso di sicurezza nell'affrontare situazioni di comportamenti ostili (Cahill, 2008). Il fatto che l'amministrazione dell'ospedale fornisca agli operatori formazione, genera un aumento di fiducia nella struttura organizzativa, determinando anche l'aumento del numero dei casi di aggressione denunciati (Deans, 2004). Secondo alcuni, questo tipo di formazione dovrebbe accompagnare l'infermiere in tutto l'arco della vita lavorativa, a partire dagli studi universitari, per poi proseguire con programmi di formazione continua nelle singole sedi lavorative (Deans, 2004).

Per diversi autori (Kwok et al. 2006; Rodgers et al. 2004; Winstanley et al. 2004; Crilly et al. 2004) il problema necessita di ulteriori studi, e questo pensiero viene condiviso dal Ministero della Salute italiano nella raccomandazione n. 8 del 2007 (Ministero della Salute, 2007). Il Ministero identifica una serie di atti ritenuti fondamentali per riuscire a prevenire il fenomeno delle violenze contro gli operatori sanitari, che prevedono elaborazione di programmi di prevenzione, analisi delle situazioni lavorative, definizione di misure di prevenzione e controllo, ed infine formazione rivolta a tutto il personale, anche a livello manageriale.

La segnalazione degli incidenti, viene vista come un punto fondamentale nell'affrontare il fenomeno (Zampieron et al. 2010; Crilly et al. 2004; Jenkinset al. 1998; Crabbe et al. 2002; Opie et al. 2010), assieme all'istituzione di specifici registri per le diverse tipologie di violenze.

Luck e collaboratori (2007) nel loro studio usano un approccio diverso, proponendo l'osservazione di componenti comportamentali facilmente identificabili, che indichino un potenziale atteggiamento ostile e aggressivo. Gli autori hanno individuato cinque comportamenti tipici di situazioni a rischio di violenze, che vengono indicate con l'acronimo STAMP. Gli atteggiamenti ritenuti indicativi sono: sguardo fisso, tono di voce, ansia, borbottio, camminare avanti e indietro. Un lavoro analogo, svolto utilizzando la metodologia Delphi, è stato condotto da Wilkes et al. (2010) per costruire uno strumento predittivo di comportamenti violenti nei contesti di emergenza.

Un cambiamento delle strutture fisiche interne, intese come organizzazione degli spazi, viene proposto da Cembrowicz et al. (1992), Lavoie et al. (1988), Pich et al. (2011) e Gilchrist et al. (2011), i quali promuovono anche l'adozione di telecamere a circuito chiuso interne e l'aumento della presenza fissa di agenti di sicurezza. In Italia si chiede che gli infermieri, a fronte del loro ruolo di operatori di prima linea, siano coinvolti nelle ristrutturazioni e organizzazioni degli spazi dei DEA (Becattini et al. 2007).

Le soluzioni finora prospettate, sono tutte volte alla prevenzione o alla gestione degli eventi aggressivi, e solo pochi lavori infatti trattano della prevenzione delle conseguenze psicologiche delle violenze. Uno studio riporta che nel $60 \%$ dei casi dopo un evento di violenza, non viene proposto alla vittima nessun debriefing, mentre, a volte, quelli eseguiti sono stati tenuti in modo inadeguato e da personale non preparato (Ross-Adjie et al. 2007).

Gli infermieri inoltre ritengono che i debriefing dovrebbero essere più informali e personali per evitare di sentirsi sotto accusa (Pich et al. 2011). Talvolta, sembra che il supporto fornito dalle amministrazioni alle vittime duri troppo poco tempo (Lyneham, 2000).

\section{DISCUSSIONE}

La letteratura analizzata, benché proveniente da diverse realtà internazionali, vede di gran lunga i paesi di lingua anglofona come maggiori autori di ricerche al riguardo. Questo denota una più grande sensibiliz- 
zazione al problema delle aggressioni da parte delle nazioni che hanno avuto nella professione infermieristica forti influenze da parte del modello anglosassone, o che portano avanti un'ingente produzione scientifica infermieristica come gli USA.

In ogni caso dalla lettura degli articoli emerge come il problema delle aggressioni agli infermieri di pronto soccorso sia equamente diffuso in maniera molto importante e preoccupante, e mostra come la quasi totalità degli operatori studiati sia venuta in contatto almeno una volta con una qualsiasi tipologia di violenza, la più frequente delle quali risulta essere l'abuso verbale, confermando l'ampia estensione di un fenomeno con importanti risvolti negativi in ambito lavorativo. I colleghi che vivono in ambienti così a rischio, lavorano con un senso di paura ed insicurezza continui (Opie et al. 2010), tali da essere in alcuni casi la causa dell'allontanamento dal Pronto Soccorso (Opie et al. 2010). Le aggressioni fisiche non impattano solamente sul corpo delle vittime, ma lasciano sequele psicologiche che possono diminuire anche in termini di sicurezza le performance lavorative degli operatori (Gates et al. 2011; Tang et al. 2007).

Un alto numero di studi non riporta chiaramente chi siano gli autori delle violenze, o comunque li indicano in maniera generica in ordine di importanza senza fornire dati numerici (Eslamian et al. 2010; Pich et al. 2011; Deans, 2004; Kwok et al. 2006; Crilly et al. 2004; Lyneham, 2000, Rose, 1997; Schnieden et al. 1995; Cahill 2008; Crabbe et al. 2002; Opie et al. 2010; Adeb-Saeedi, 2002). Questo, indica il percorso da seguire per ricerche future. E' necessario spostare l'attenzione maggiormente sulle caratteristiche dei perpetratori/aggressori, associandoli alle diverse tipologie di violenze. Inoltre, andrebbero maggiormente indagate le diverse reazioni delle vittime alle aggressioni, le conseguenze sulla loro salute psicofisica e sociale, l'impatto sulla performance lavorativa, e l'efficacia delle soluzioni prospettate anche nel lungo termine.

Nonostante l'attenzione che i media stanno dando al problema sempre più consistentemente, occorre migliorare l'adesione ai sistemi di report, dove presenti, per favorire le attività di osservatorio basati su database/registri istituiti ad hoc.

\section{BIBLIOGRAFIA}

Adeb-Saeedi J. (2002). Stress amongst emergency nurses. Australian Emergency Nursing Journal, 5 (2), 19-24

Bambi, S, Scarlini, D, Becattini, G, Alocci, P, \& Ruggeri, M. (2011). Characteristics of patients who leave the ED triage area without being seen by a doctor: a descriptive study in an urban level II Italian
University Hospital. Journal of Emergency Nursing, 37 (4), 334-40

Becattini, G., Bambi, S., Palazzi, F., \& Lumini, E (2007). Il fenomeno delle aggressioni agli operatori di Pronto Soccorso: la prospettiva italiana. XXVI ${ }^{\circ}$ Congresso Nazionale ANIARTI - Rimini, 24-26 Ottobre 2007. Atti del Congresso.

Cahill, D.(2008).The effect of ACT-SMART on nurses' perceived level of confidence toward managing the aggressive and violent patient. Advanced Emergency Nursing Journal, 30 (3), 252-70

Cembrowicz, S.P., \& Shepherd, J.P. (1992). Violence in the Accident and Emergency Department. Medicine, Science and the Law, 32 (2), 118-22

CDC Workplace Safety and Health (2002). Occupational hazards in hospital. Data accesso 17 Marzo, 2012. Da http://www.cdc.gov/niosh/pdfs/2002-101.pdf

Crabbe, J., Alexander, D.A., Klein, S., Walker, S., \& Sinclair J. (2002). Dealing with violent and aggressive patients: at what cost to nurses? Irish Journal of Psychological Medicine, 19 (4), 121-4

Crilly, J., Chaboyer, W., \& Creedy D. (2004). Violence towards emergency department nurses by patients. Accident \& Emergency Nursing, 12 (2), 67-73

Deans, C. (2004). The effectiveness of a training program for emergency department nurses in managing violent situations. Australian Journal of Advanced Nurses, 21 (4), 17-22

Department of Health Western Australia (2004). Prevention of workplace aggression and violence. Data accesso 18 Marzo, 2012. Da http://www.nursing.health.wa.gov.au/docs/reports/ WorkplaceAggression_Violence.pdf

Eslamian, J., Fard, S.H., Tavakol, K., \& Yazdani M. (2010). The effect of anger management by nursing staff on violence rate against them in the emergency unit. Iranian Journal of Nursing and Midwifery Research, 15 (Special Issue), 337-342

Gates, D.M., Gillespie, G.L., \& Succop, P. (2011). Violence against nurses and its impact on stress and productivity. Nursing Economics, 29 (2), 59-67

Gilchrist, H., Jones, S.C., \& Barrie, L. (2011). Experiences of emergency department staff: Alcoholrelated and other violence and aggression. Australasian Emergency Nursing Journal, 14 (1), 9-16

Gillespie, G.L., Gates, D.M., Miller, M., \& Howard, P.K. (2010). Violence against healthcare workers in a pediatric emergency department. Advanced Emergency Nursing Journal, 32 (1), 68-82

Kansagra, S.M., Rao, S.R., Sullivan, A.F., Gordon, J.A., Magid, D.J., Kaushal, R., Camargo, C.A. Jr, \& Blumenthal, D. (2008). A survey of workplace violence across 65 U.S. emergency departments. Academic Emergency Medicine, 15 (12), 1268-74

Kwok, R.P., Law, Y.K., Li, K.E., Ng, Y.C., Cheung, M.H., Fung, V.K., Kwok, K.T., Tong, J.M., Yen, P.F., \& 
Leung, W.C. (2006). Prevalence of workplace violence against nurses in Hong Kong. Hong Kong Medical Journal, 12 (1): 6-9

Jenkins, M.G., Rocke, L.G., McNicholl, B.P., \& Hughes D.M. (1998). Violence and verbal abuse against staff in accident and emergency departments: a survey of consultants in the UK and the Republic of Ireland. Journal of Accident and Emergency Medicine, 15 (4), 262-5

Lavoie, F.W., Carter, G.L., Danzl, D.F., \& Berg, R.L. (1988). Emergency department violence in United States teaching hospitals. Annals of Emergency Medicine, 17 (11), 1227-33

Luck, L., Jackson, D., \& Usher, K. (2007). STAMP: components of observable behaviour that indicate potential for patient violence in emergency departments. Journal of Advanced Nursing, 59 (1), 11-9

Luck, L., Jackson, D., \& Usher, K. (2008). Innocent or culpable? Meanings that emergency department nurses ascribe to individual acts of violence. Journal of Clinical Nursing, 17 (8), 1071-8

Lyneham, J. (2000). Violence in New South Wales emergency departments. Australian Journal of Advanced Nurses, 18 (2), 8-17

Ministero della Salute (2007). Raccomandazione n. 8 Raccomandazione per prevenire gli atti di violenza a danno degli operatori sanitari. Data accesso 16 Marzo, 2012. Da http://www.salute.gov.it/ imgs/C_17_pubblicazioni_721_allegato.pdf

Ministero della Salute (2009). Monitoraggio degli eventi sentinella. Data accesso 16 Marzo, 2012. Da http://www.salute.gov.it/qualita/paginaInternaQualita. jsp?id=238\&menu=sicurezza

Opie, T., Lenthall, S., Dollard, M., Wakerman, J., MacLeod, M., Knight, S., Dunn, S., \& Rickard, G. (2010). Trends in workplace violence in the remote area nursing workforce. Australian Journal of Advanced Nursing, 27 (4), 18-23

Perrone, S. (1999). Violence in the workplace. Australian Institute of Criminology Research and Public Policy Series No. 22. Issue overviews. Data accesso 18 Marzo, 2012. Da http://www.aic.gov.au/documents /4/1/0/\%7B410FD288-AD 82-4E4E-A6FEC7C30D283528\%7Dfull_report.pdf

Pich, J., Hazelton, M., Sundin, D., \& Kable, A. (2011) Patient-related violence at triage: A qualitative descriptive study. International Emergency Nursing,
19 (1), 12-19

Pinar, R., \& Ucmak, F. (2011). Verbal and physical violence in emergency departments: a survey of nurses in Istanbul, Turkey. Journal of Clinical Nursing, 20 (3-4), 510-7

Rodger, M., Hills, J., \& Kristjanson, L. (2004). A Delphi study on research priorities for emergency nurses in Western Australia. Journal of Emergency Nursing, 30 (2), 117-25

Rose, M. (1997) A survey of violence toward nursing staff in one large Irish Accident and Emergency Department. Journal of Emergency Nursing, 23 (3), 214-9

Ross-Adjie, G.M., Leslie, G., \& Gillman, L. (2007). Occupational stress in the ED: what matters to nurses? Australasian Emergency Nursing Journal, 10 (3), 117-23

Ryan, D., \& Maguire, J. (2006). Aggression and violence a problem in Irish Accident and Emergency departments? Journal of Nursing Management, 14 (2), 106-15

Schnieden, V., \& Marren-Bell, U. (1995). Violence in the accident and emergency department. Accident \& Emergency Nursing, 3 (2), 74-8

Tang, J.S., Chen, C.L., Zhang, Z.R., \& Wang, L. (2007) Incidence and related factors of violence in emergency departments--a study of nurses in southern Taiwan. Journal of the Formosan Medical Association, 106 (9), 748-58

The Joint Commission (2010). Preventing violence in the health care setting. Issue 45, June 3. Data accesso 16 Marzo, 2012. Da http://www.jointcommission.org /assets/1/18/SEA_45.PDF

United States Department of Labor, Occupational Safety and Health Administration (2012). Workplace Violence. Data accesso 17 Marzo, 2012. da http://www.osha.gov/SLTC/workplaceviolence/

Wilkes, L., Mohan, S., Luck, L., Jackson, D. (2010). Development of a violence tool in the emergency hospital setting. Nurse Researcher, 17 (4), 70-82

Winstanley, S., \& Whittington, R. (2004). Aggression towards health care staff in a UK general hospital: variation among professions and departments. Journal of Clinical Nursing, 13 (1), 3-10

Zampieron, A., Galeazzo, M., Turra, S., \& Buja A (2010). Perceived aggression towards nurses: study in two Italian health institutions. Journal of Clinical Nursing, 19 (15-16), 2329-41 